\title{
High volume image guided injections and structured rehabilitation in shoulder impingement syndrome: a retrospective study
}

\author{
Sarah Morton 1 \\ Otto Chan² \\ Asser Ghozlan 1 \\ Jessica Price ${ }^{1}$ \\ John Perry ${ }^{1,2}$ \\ Dylan Morrissey ${ }^{1,2,3}$
}

1 Centre for Sports and Exercise Medicine William Harvey Research Institute Queen Mary University of London Mile End Hospital, London, UK

2 BMI London Independent Hospital, London, UK

3 Physiotherapy Department, Bart's Health NHS Trust, London, UK

Corresponding author:

Dylan Morrissey

Centre for Sports and Exercise Medicine William Harvey Research Institute Queen Mary University of London Mile End Hospital

Bancroft road, E1 4DG London, UK

E-mail: d.morrissey@gmul.ac.uk

\section{Summary}

Background: the aim was to establish the effect of a high volume-image guided injection and structured rehabilitation (HVIGI\&SR) on both pain and function in shoulder impingement syndrome (SIS). Methods: 44 participants treated between January 2008 and January 2012 with a >3 month history of recalcitrant ultrasound-confirmed SIS were sent a retrospective questionnaire. All participants had received a HVIGI under ultrasound-guidance consisting of $20 \mathrm{mls}$ of Marcaine with $50 \mathrm{mg}$ of hydrocortisone, followed by a period of physiotherapist-led rehabilitation. The validated Shoulder Pain and Disability Index (SPADI) score was used to establish the change in the score between 1 week pre-injection and 3 weeks post-injection, along with an 11-point pain scale.

Results: $59 \%$ of participants responded. There was a clinically and statistically significant decrease in the SPADI score of $58.7 \pm 29.9(p<0.01)$. $76 \%$ of participants had an improvement in their score of over $50 \%$ from their initial score. There was a clinically and statistically significant improvement in pain of $5.19 \pm 2.62(p<0.01)$ on the numerical rating scale of pain.
Conclusion: HVIGI\&SR should be considered for short-term treatment of SIS as it showed a significant improvement in both pain and function. A prolonged period of physiotherapist-led rehabilitation can then be undertaken for long term benefits.

KEY WORDS: injection, rotator cuff, SPADI, ultrasound.

\section{Introduction}

Approximately $50 \%$ of the population has at least one episode of shoulder pain every year and the cost to the economy is significant ${ }^{1-4}$. The prevalence of shoulder impingement syndrome is estimated at $43.7 \%$ in upper arm sports, such as swimming or tennis ${ }^{1}$. Shoulder impingement syndrome is an umbrella term that encompasses numerous conditions such as partial thickness rotator cuff tears, rotator cuff tendinosis and calcific tendinitis 5,6 . Recent work has suggested that in fact "impingement" does not satisfactorily describe the pathology underlying the pain and it should in fact be called "rotator cuff disease"7. However, with more detail in the literature relating to shoulder impingement syndrome this is the term that will be used in this manuscript.

Shoulder impingement syndrome causes both pain and difficulty in performing everyday activities ${ }^{1,6}$. Looking at partial thickness rotator cuff tears alone it is recognised that patients can present with mild pain, chronic pain or shoulder instability ${ }^{8}$. According to Bigliani and Levine (1997) treatment falls broadly into conservative and surgical management ${ }^{5}$. Conservative treatment, recommended as first line treatment, includes non-steroidal anti-inflammatory drugs, physiotherapy, extracorporeal shock wave therapy and subacromial steroid injections $s^{5,6,9-11}$. Indeed it is recognised that partial tears may develop into fullthickness tears if left untreated ${ }^{12}$. Today the most common surgical technique used is arthroscopic subacromial depression, although the evidence for both conservative and surgical treatment is limited and no agreement exists within the literature as to what is the best surgical technique ${ }^{6,8}$. Complications of surgical repair include re-tearing and non-healing with pain therefore recurring ${ }^{12}$.

Smidt and Greenhave suggested that there is limited benefit of any conservative treatments for surgical impingement syndrome ${ }^{13}$. However, it has been shown that the addition of a physiotherapy regime to a corticosteroid injection is beneficial and provides a faster 
improvement in shoulder range of motion, as well as improving pain ${ }^{14}$. A recent systematic review showed shock wave therapy to improve shoulder function and pain, as well as dissolving calcifications in calcific tendinitis ${ }^{9}$.

Chan et al. (2008), Crisp et al. (2008), Morton et al. (2014), and Humphrey et al. (2010) have shown that high volume image-guided injections are effective in treating chronic Achilles and patellar tendinopathy 15-18. It is also not only in tendinopathy that high volume image guided injections have been shown to be effective ${ }^{19,20}$. Ultrasound guided injections of corticosteroids in shoulder pain have been shown to be more effective than blind injections ${ }^{21}$. The aim of this study was therefore to outline the novel use of a high volume image-guided injection in shoulder impingement syndrome followed by a physiotherapist-led rehabilitation regime and establish the effect it has on pain and function. Our alternative hypothesis was that there would be an improvement in both pain and function that was both statistically and clinically significant.

\section{Materials and methods}

Forty four patients who each received a single high volume image-guided injection and structured rehabilitation programme (HVIGI\&SR) to their shoulder at one specialist musculoskeletal (MSK) centre between January 2008 and January 2012 were retrospectively sent a questionnaire for both pre and post injection. All of these participants had been referred to the specialist MSK centre having failed initial rehabilitation and having seen at least one other clinician prior to their referral.

The questionnaire included both the SPADI (shoulder pain and disability index), a validated questionnaire for both shoulder pain and function which grades a normal shoulder as 0 and maximally affected as 130 , and an 11 point numerical pain rating scale with 0 as normal and 10 as maximal pain ${ }^{22}$. Whilst it is recognised in the literature that an accepted definitive scoring system for shoulder pathology treatment is lacking the SPADI was chosen to describe pain and function as it has good psychometric properties ${ }^{23}$. The primary outcome measure was therefore the change in the SPADI score from 1 week pre injection to 3weeks post injection. Our study was therefore considered a retrospective case series design.

Patients aged between 18-70 years were included if they had at least 3 months history of shoulder pain and loss of function. All participants had been evaluated by a minimum of one clinician and received either physiotherapy or another intervention without satisfactory improvement prior to their HVIGI\&SR. Failure of initial conservative management was considered if there was deemed to be sub-optimal improvement or continued severe pain that prevented rehabilitation.

The diagnosis of shoulder impingement syndrome was based on a combination of clinical history, clinical examination and imaging. This was performed by a stable, cohesive team of experienced clinicians with an agreed care pathway to exclude other differential diagnoses (f.e. the apprehension test was used to rule out instability and the empty can test to exclude a rotator cuff tear). Data relating to how many participants were excluded secondary to this is unavailable.

Any frank tears and tendinopathic changes such as bursal thickening or neovascularization were excluded using an ultrasound scan, performed by a MSK radiologist; no participants were found to have frank tears on ultrasound prior to the injection. Participants were excluded if they previously had shoulder surgery or suffered from any significant systemic disease, such as diabetes mellitus. Non-responders, who had not returned the questionnaire within five weeks, were followed up via telephone six weeks after initially receiving the questionnaire.

\section{High volume image guided injection technique}

All ultrasound scanning and interventions were carried out by a single experienced radiologist using the same ultrasound scanner throughout (Elegra; Siemens, Erlangen, Germany). A $13 \mathrm{MHz}$ transducer along with power Doppler imaging was used to guide all injections. The patients were seated with the ipsilateral arm hanging down and the probe placed in the coronal plane over the lateral end of the acromion. A 21G green needle was inserted in the plane directed towards the anaechoic space of subacromial/subdeltoidbursae (Fig. 1). $20 \mathrm{mls}$ of $0.5 \%$ marcaine mixed with $50 \mathrm{mg}$ of hydrocortisone was injected under real-time ultrasound guidance using an aseptic technique.

The participants were then referred for a standardised physiotherapist-led rehabilitation regime by a consultant physiotherapist; this included immediate scapular retraining to optimise scapular positioning when the participant lifted their arm $^{24}$. Also in the first week emphasis was placed on achieving full shoulder girdle range of movement with good scapula-humeral rhythm. Following on from this, and once the consul-

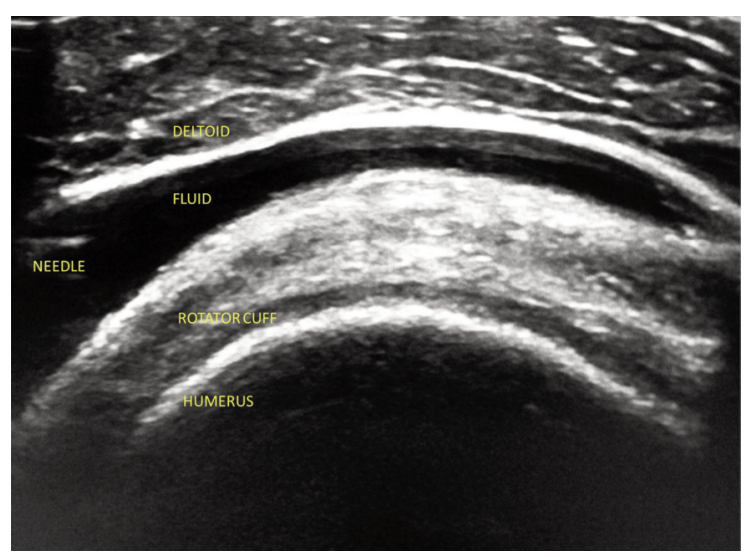

Figure 1. Image of a high volume image-guided injection in shoulder impingement syndrome. 
tant physiotherapist felt the movement patterns were corrected satisfactorily, functional strength deficits were addressed based on an individual participant's presentation and sports or activity related requirements over a six-week period. In order to move forward to loaded rehabilitation the participant had to demonstrate good scapular control in each plane under the load of a straight arm, with a particular emphasis in maintaining upward rotation and posterior tilt. These latter two movements have been shown to be commonly associated with impingement ${ }^{25}$.

\section{Data analysis}

Data were analysed using SPSS version 20 (IBM, USA) with a paired t-test used to analyse both the pre and post-injection SPADI scores and pain scale scores. Statistical significance was set at $p<0.05$.

\section{Ethical considerations}

Ethical clearance was obtained. Consent was obtained via the questionnaire. The study meets the ethical guidance and standards as laid out by Padulo et al. $(2013)^{26}$.

\section{Results}

Twenty-six out of forty four participants (59\%) who were initially sent the questionnaire returned it fully completed. Participant characteristics are shown in Table 1.

Table 1. Participant characteristics.

\begin{tabular}{ll}
\hline Number of participants & 26 \\
Mean Age $( \pm S D)$ & $51.4 \pm 11.3$ \\
Sex & 15 females: 11 males \\
Shoulder injected & 13 left: 11 right: 2 unknown \\
\hline
\end{tabular}

SD; Standard Deviation
There was a statistically significant decrease in the overall SPADI score of $58.7 \pm 29.9(t=9.94, p<0.01$, $95 \% \mathrm{Cl}: 46.2-70.3$ ) from $86.5 \pm 27.6$ to $28.2 \pm 26.1$. Over $76 \%$ of participants had an improvement in their score of over $50 \%$. A decrease in the score greater than 18.1 is consi dered a clinically significant improvement ${ }^{27}$. The individual results are shown in Figure 2 .

There was a statistically significant decrease of $5.19 \pm 2.62$ in the mean pain component of the SPADI from $7.81 \pm 1.63$ to $2.62 \pm 2.62(\mathrm{t}=10.1, \mathrm{p}<0.01,95 \% \mathrm{Cl}$ : 4.13-6.25). No complications were reported by any of the participants. $50 \%$ of participants stated their symptoms were back to normal, or strongly agreed their symptoms had improved within the 3 weeks. Only $12 \%$ felt their symptoms had not improved. $38 \%$ felt they had neither improved nor worsened within the three-week period.

\section{Discussion}

This is the first study into high volume image-guided injections in shoulder impingement syndrome. A significant improvement in both the SPADI score and pain scale were shown. The mean decrease in the SPADI score of 58.9 is considered clinically significant, based on a minimal important difference of 13.2 and a minimum detectable change of $18.1^{27} .67 \%$ of participants had a greater than $50 \%$ improvement in their SPADI score which is comparable to similar injections used in shoulder impingement syndrome, such as in the study by Hollingworth et al. $(1983)^{28}$. The results appear to show a better short-term improvement in comparison to when only non-steroidal anti-inflammatory drugs and physiotherapy were used $^{29}$. As surgical treatment has no evidence to suggest it is superior to conservative treatment in shoulder impingement syndrome, it is likely that a high volume image-guided injection is comparable without the additional risks of surgery ${ }^{30}$. Other injection therapies have also shown similar effectiveness in short term follow-up in double blind trials, as has exercise

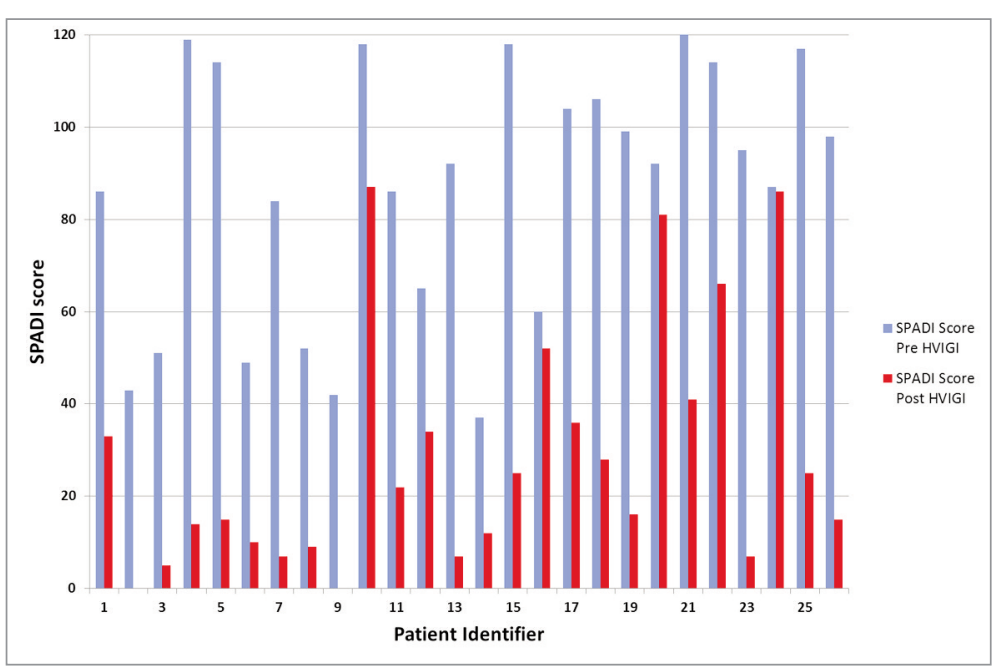

Figure 2. Individual changes in SPADI scores pre- and post-high volume imageguided injection. 
and manual therapy alone ${ }^{31,32}$. It is therefore highly likely that HVIGI\&SR has a similar benefit in the short term for SIS as was shown by Carette et al. (2003) in adhesive capsulitis when they used an intra-articular corticosteroid and physiotherapy regime in comparison to a placebo group ${ }^{14}$. However prospective casecontrol studies are required to confirm this.

The results are also comparable to the findings in both Achilles tendinopathy and patellar tendinopathy ${ }^{15,18}$. As described by Chan et al. (2008), the mechanism of HVIGI\&SR action remains unproven, but it has been logically suggested that large volumes of fluid within a confined space disrupt neo-vessels and the accompanying nerve supply, which are associated with tendinopathy, by breaking or stretching the vessels whilst also mobilising adhesions ${ }^{15,33,34}$. The mechanism of action within the shoulder may be similar if tendinopathy is present, and we typically observed increased range of movement suggesting soft tissue mobilisation. However, as seen in both greater trochanter pain syndrome and medial collateral ligament injuries, it may be that there is disruption of scar tissue or separation of tissues and mobilisation of adhesions ${ }^{19,20}$. This is an area where further research is required, possibly using further imaging or surgical confirmation.

\section{Study limitations}

The follow up time of three weeks in this study was relatively short and further research is required to establish the effect over a longer period of time, preferably with prospective data. The participants did not report any additional treatments during the three weeks, suggesting the effect was due to the high volume image guided injection and subsequent physiotherapy regime only. It is likely that the addition of the physiotherapy will result in the long term gains seen in previous studies, although this would require further follow up in the future ${ }^{14}$. Due to the fact all the participants had pain for more than three months prior to the injection it is unlikely that the resolution in symptoms seen was due to time alone; however prospective casecontrolled studies are required to ensure this. We should also consider that shoulder impingement syndrome is an umbrella term and that some diagnoses within that may respond better than others, an area that should be explored further in the future.

The mean age of the participants was $51.4 \pm 11.3$, which although is useful for this population, means that its effectiveness in younger participants, especially within professional sport, is not clear. This is therefore another area for future research and follow-up.

As our study was a retrospective case series it was possibly prone to recall bias and this may impact on the results. However all the participants had experienced pain for at least 3 months prior to their HVIGI\&SR and therefore it is likely that any improvement from the injection would be noticeable and memorable and therefore less likely to have impacted upon the results. Furthermore, as this is a first report, the data should not be regarded as definitive proof of effect but is valuable as a guide to further research and clinical innovation.

The study was also carried out over a period of four years and therefore there was an apparently high 'lost-to-follow-up' rate of $41 \%$. The population group studied included a high number of tertiary referrals and local inner London patients including city-workers with quite a high change of residence annually. For these reasons, we do not believe the loss to follow up ratio is either excessively high or likely to represent a systematic effect. Further, questionnaire response rates in such patient groups are rarely much higher unless the outcome measure is administered at a patient contact, which renders such long term follow-up impossible.

\section{Future research}

As described above further research is required to determine the mechanism of HVIGI\&SR in shoulder impingement syndrome. More work is also required to establish the duration of the effect and the addition of further ongoing physiotherapy. Future prospective case-controlled studies should ensure that measures are taken to optimize response and minimize concerns due to low response rates. Comparative studies would allow the effectiveness in comparison to other treatment modalities be made, as well as establishing patients' experience.

\section{Conclusion}

High volume image-guided injections followed by a physiotherapist-led rehabilitation regime have shown an improvement in both pain and function in the short term treatment of shoulder impingement syndrome where conservative measures have already failed. This is a first report of a novel technique that shows promising results.

\section{References}

1. Lo YP, Hsu YC, Chan KM. Epidemiology of shoulder impingement in upper arm sports events. Br J Sports Med. 1990;24: 173-177.

2. Meislin RJ, Sperling JW, Stitik TP. Persistent shoulder pain: epidemiology, pathophysiology, and diagnosis. Am J Orthop (Belle Mead, NJ). 2005;34:5-9.

3. Lewis JS. Rotator cuff tendinopathy. Br J Sports Med. 2009;43:236-241.

4. van der Heijden G. Shoulder disorders: a state-of-the-art review. Baillieres Best Pract Res Clin Rheumatol. 1999;13:287-309.

5. Bigliani LU, Levine WN. Subacromial impingement syndrome. J Bone Joint Surg Am. 1997;79:1854-1868.

6. Koester MC, George MS, Kuhn JE. Shoulder impingement syndrome. Am J Med. 2005;118:452-455.

7. McFarland EG, Maffulli N, Del Buono A, Murrell GA, GarzonMuvdi J, Petersen SA. Impingement is not impingement: the case for calling it "Rotator Cuff Disease". Muscles Ligaments Tendons J. 2013;3:196-200. 
8. Franceschi F, Papalia R, Del Buono A, Maffulli N, Denaro V. Repair of partial tears of the rotator cuff. Sports Med Arthrosc. 2011;19: 401-408.

9. Ioppolo F, Tattoli M, Di Sante L, Venditto T, Tognolo L, Delicata $\mathrm{M}$, et al. Clinical improvement and resorption of calcifications in calcific tendinitis of the shoulder after shock wave therapy at 6 months' follow-up: a systematic review and metaanalysis. Arch Phys Med Rehabil. 2013;94:1699-1706.

10. Mouzopoulos G, Stamatakos M, Mouzopoulos D, Tzurbakis M. Extracorporeal shock wave treatment for shoulder calcific tendonitis: a systematic review. Skeletal Radiol. 2007;36:803-811.

11. Longo UG, Franceschi F, Berton A, Maffulli N, Droena V. Conservative treatment and rotator cuff tear progression. Med Sport Sci. 2012;57:90-99.

12. Osti L, Del Buono A, Maffulli N. Rotator cuff repair: imaging success and clinical results may not correspond. Orthopedics. 2014;37:17-18.

13. Smidt N, Green S. Is the diagnosis important for the treatment of patients with shoulder complaints? Lancet. 2003;362:18671868.

14. Carette S, Moffet FN, Tardif J, Bessette L, Morin F, Fremont P, et al. Intraarticular corticosteroids, supervised physiotherapy, or a combination of the two in the treatment of adhesive capsulitis of the shoulder. Arthritis Rheum. 2003;48:829-838.

15. Chan O, O'Dowd D, Padhiar N, Morrissey D, King J, Jalan R, Maffulli N, Crisp T. High volume image guided injections in chronic Achilles tendinopathy. Disabil Rehabil. 2008;30:16971708.

16. Crisp T, Khan F, Padhiar N, Morrissey D, King J, Jalan R, Maffulli $\mathrm{N}$, Chan $\mathrm{O}$. High volume ultrasound guided injections at the interface between the patellar tendon and Hoffa's body are effective in chronic patellar tendinopathy: A pilot study. Disabil Rehabil. 2008;30:1625-1634.

17. Humphrey J, Chan O, Crisp T, Padhiar N, Morrissey D, Twycross-Lewis R, et al. The short-term effects of high volume image guided injections in resistant non-insertional Achilles tendinopathy. J Sci Med Sport. 2010;13:295-298.

18. Morton S, Chan O, King J, Perry D, Crisp T, Maffulli N, Morrissey D. High volume image-guided injections for patellar tendinopathy: a combined retrospective and prospective case series. Muscles Ligaments Tendons J. 2014;4:214-219.

19. Morton S, Chan O, Price J, Pritchard M, Crisp T, Perry J, Morrissey $\mathrm{D}$. High volume image-guided injections and structured rehabilitation improve greater trochanter pain syndrome in the short and medium term: a combined retrospective and prospective case series. Muscles Tendons Ligaments J. 2015;5:73-87.

20. Drumm O, Chan O, Malliaras P, Morrissey D, Maffulli N. Highvolume image-guided injection for recalcitrant medial collatera ligament injuries of the knee. Clin Radiol. 2014;69:e211-215.

21. Soh E, Li WY, Ong KO, Chen W, Bautista D. Image-guided versus blind corticosteroid injections in adults with shoulder pain: A systematic review. BMC Musculoskelet Disord. 2011:12:137.

22. Williams JW Jr, Holleman DR Jr, Simel DL. Measuring shoulder function with the Shoulder Pain and Disability Index. J Rheumatol. 1995;22:727-732.

23. Longo UG, Vasta S, Maffulli N, Denaro V. Scoring systems for the functional assessment of patients with rotator cuff pathology. Sports Med Arthrosc. 2011;19:310-320.

24. Worsley P, Warner M, Mottram S, Gadola S, Veeger HEJ, Hermens $\mathrm{H}$, et al. Motor control retraining exercises for shoulder impingement: effects on function, muscle activation, and biomechanics in young adults. J Shoulder Elbow Surg. 2013;22: E11-E9.

25. Ludewig PM, Cook TM. Alterations in shoulder kinematics and associated muscle activity in people with symptoms of shoulder impingement. Phys Ther. 2000;80:276-291.

26. Padulo J, Oliva F, Frizziero A, Maffulli N. Muscles, Ligaments and Tendons Journal. Basic principles and recommendations in clinical and field science research. MLTJ. 2013;4:250-252.

27. Schmitt JS, Di Fabio RP. Reliable change and minimum important difference (MID) proportions facilitated group responsiveness comparisons using individual threshold criteria. J Clin Epidemiol. 2004;57:1008-1018.

28. Hollingworth GR, Ellis RM, Hattersley TS. Comparison of injection techniques for shoulder pain - results of a double blind, randomized study. BMJ (Clin Res Ed). 1983;287:1339-1341.

29. Morrison DS, Frogameni AD, Woodworth P. Non-operative treatment of subacromial impingement syndrome. J Bone Joint Surg Am. 1997;79A:732-737.

30. Gebremariam L, Hay EM, Koes BW, Huisstede BM. Effectiveness of Surgical and Postsurgical Interventions for the Subacromial Impingement Syndrome: A Systematic Review. Arch Phys Med Rehabil. 2011;92:1900-1913.

31. Dogu B, Yucel SD, Sag SY, Bankaoglu M, Kuran B. Blind or ultrasound-guided corticosteroid injections and short-term response in subacromial impingement syndrome: a randomized, double-blind, prospective study. Am J Phys Med Rehabil. 2012;91:658-665.

32. Crawshaw DP, Helliwell PS, Hensor EM, Hay EM, Aldous SJ, Conaghan PG. Combined Treatment with Corticosteroid Injection Plus Exercise and Manual Therapy Was Similar to Exercise and Manual Therapy Alone for Shoulder Pain at 12 Weeks. J Bone Joint Surg Am. 2011;93A:971.

33. Lewis JS, Raza SA, Pilcher J, Heron C, Poloniecki JD. The prevalence of neovascularity in patients clinically diagnosed with rotator cuff tendinopathy. BMC Musculoskelet Disord. 2009;10:163.

34. De Jonge S, Warnaars JL, De Vos RJ, Weir A, van Schie HT, Bierma-Zeinstra SM, et al. Relationship between neovascularization and clinical severity in Achilles tendinopathy in 556 paired measurements. Scand J Med Sci Sports. 2014;24:773-778. 